\title{
La función explicativa de los modelos en biología
}

\section{The explanatory function of models in biology}

\author{
ANTONIO DIÉGUEZ \\ Departamento de Filosofía. Universidad de Málaga (España)
}

Recibido: 30-1-2013

Aprobado definitivamente: 12-3-2013

\section{RESUMEN}

Los modelos científicos son recursos explicativos fundamentales en la ciencia, y particularmente en aquellas ciencias en las que es dudoso que podamos contar con leyes científicas genuinas, como es el caso de la biología (y de las ciencias sociales). La cuestión de cómo explican los modelos ha despertado una gran atención en las últimas décadas y, sin embargo, sigue siendo una cuestión controvertida. Hay muchos tipos de modelos y no es de extrañar, por tanto, que puedan proporcionar explicaciones de los fenómenos de formas muy diversas. Si se puede señalar un rasgo común a todos estos modos diferentes de explicar, es el hecho de que los modelos nos ofrecen una mejor comprensión de los fenómenos. Se argumenta en este trabajo que la noción de 'comprensión' aquí implicada no es irremediablemente subjetiva.

\section{PALABRAS CLAVE \\ MODELOS BIOLÓGICOS, EXPLICACIÓN CIENTÍFICA, COMPRENSIÓN, LEYES CIENTÍFICAS}

\footnotetext{
ABSTRACT

Scientific models are basic explanatory resources in science. This explanatory function is especially relevant in biology and social sciences, where it is doubtful the existence of genuine scientific laws. How models can provide scientific explanations has been a widely debated issue 
in the past decades, but in spite of this fact it remains as a controversial one. There are many kinds of models in biology, so it is not surprising that they provide scientific explanations of phenomena in very different ways. A possible common feature among this diversity is the fact that models give us a better understanding of phenomena. It is argued in this paper that the notion of 'understanding' is not irremediably subjective.

KEYWORDS

BIOLOGICAL MODELS, SCIENTIFIC EXPLANATION, UNDERSTANDING, SCIENTIFIC LAWS

ES AMPLIAMENTE ACEPTADO que una de las funciones principales de los modelos científicos es proporcionar una explicación de los fenómenos que representan. Se dice, por ejemplo, que el modelo operón de la lactosa explica cómo funciona la regulación genética en algunas circunstancias; que el modelo Lotka-Volterra de competencia interespecífica explica los resultados posibles de la competición entre dos especies por el mismo nicho ecológico; que el modelo de Fisher de la ratio entre sexos explica por qué, en condiciones habituales, la proporción entre machos y hembras es de 1:1; que el modelo de doble hélice del ADN explica los patrones de difracción de rayos X detectados por Rosalind Franklin. Sin embargo, a pesar de algunos avances notables en las últimas décadas, la cuestión de cómo explican los modelos sigue siendo muy controvertida. ${ }^{1}$

La situación se torna particularmente apremiante en el caso de la biología, ya que, a diferencia de la física y de la química, ciencias en las que es habitual

1 Desde Braithwaite (1953), Hempel (1965), Nagel (1961) y Hesse (1966), diversos autores que han analizado el uso de modelos en la ciencia, o la explicación científica en general, han intentado dar cuenta del poder explicativo de los modelos científicos, si bien, pese a tales intentos, en el caso de los modelos aún carecemos de propuestas suficientemente generales y de amplio alcance. Por mencionar algunas de estas propuestas, uno de los primeros intentos de proporcionar un modelo general de explicación científica por medio de modelos fue la «explicación estructural» de Ernan McMullin (McMullin 1978). También hay que señalar la noción de explicación como simulacro de Nancy Cartwright (Cartwright 1983, cap. 8). Según Cartwrght, «explicar un fenómeno es encontrar un modelo que encaje (fits) en el marco básico de la teoría y que nos permita así derivar análogos para las complicadas y desordenadas leyes fenomenológicas que son verdaderas para el fenómeno» (p. 152). Es decir, de acuerdo con esta tesis, ofrecer un modelo analógico perteneciente al ámbito de una teoría equivale a ofrecer una explicación de un fenómeno. Sin embargo, Cartwright no desarrolla esta propuesta. Para una crítica, puede verse Elgin y Sober (2002). La «explicación por equilibrio» de Elliot Sober es otro intento que merece ser mencionado (cf. Sober 1983). En la corriente estructuralista, el asunto ha sido tratado con cierto detenimiento, aunque todos ellos son dependientes de la aceptación de la estricta noción de modelo propia de esta corriente (e. g. Bartelborth 2002, Forge 2002, Díez 2002 y Moulines 2004, y para una crítica, Frigg 2006). Más abajo mencionaremos algunas propuesta más recientes. 
el recurso a las leyes científicas para la elaboración de explicaciones, en biología son los modelos los que suelen emplearse como herramientas explicativas. Se ha dicho incluso que «la explicación en biología es obtenida siempre a través de la intervención directa de modelos de los fenómenos a explicar» (Leonelli 2009, p. 192). Es, pues, razonable preguntarse si lo que es válido según los diversos modelos de explicación científica, y muy particularmente el modelo de explicación por cobertura legal, es aplicable de algún modo a la explicación mediante modelos.

En este trabajo se discutirán algunos problemas en relación con la posibilidad de explicar mediante modelos en biología. Se argumentará que los modelos tradicionales de explicación científica no encajan bien con el modo en que se realiza la explicación mediante modelos. Los modelos pueden ofrecer explicaciones de formas muy diversas, entre otras razones porque hay muchos tipos de modelos, de modo que, muy posiblemente no sea factible reducir a un patrón único la explicación mediante modelos. No obstante, si hay un rasgo común a estos diferentes modos de proporcionar explicación -que, en tal caso, merece un análisis detenido-, éste es el logro de una mejor comprensión de los fenómenos (cf. de Regt et al. 2009).

Se argumentará asimismo que esta noción de comprensión no es, como a veces se objeta, irremediablemente subjetiva. Pueden proporcionarse algunos criterios justificables para establecer cuándo podemos suponer que un sentimiento subjetivo de comprensión se corresponde con una comprensión genuina. Este problema no es en el fondo más irresoluble que el problema clásico de distinguir entre conocimiento y creencia. Sin embargo, estos criterios no pueden ser los mismos para todo tipo de modelos. En particular, es necesario distinguir entre «modelos contrastantes»y «modelos representacionales».

\section{PROBLEMAS CON LA LEY}

La diversidad de modelos de explicación científica que han sido propuestos en las últimas décadas (el modelo de cobertura legal del empirismo lógico, el modelo unificacionista de Friedman y Kitcher, el modelo de Salmon de la relevancia estadística, así como su modelo de explicación causal, el modelo erotético de van Fraassen, etc.) no debe hacer perder de vista que hay algunos puntos acerca de la explicación científica en los que se da un amplio consenso. Son muchos los autores que, por ejemplo, coinciden en señalar que la explicación científica de un fenómeno consiste en proporcionar una respuesta a una pregunta por-qué y que esa respuesta debe incluir por lo general una identificación de la causa del fenómeno o, al menos, la incorporación del fenómeno a una estructura de relaciones causales. Muchos piensan también que una explicación científica 
debe tomar la forma de un argumento, en el que el fenómeno a explicar ha de ser derivado de una serie de premisas sujetas a ciertas condiciones restrictivas. ${ }^{1}$

Por otro lado, aunque en esto hay varios disidentes notables, ${ }^{2}$ se sostiene habitualmente que una explicación científica debe incluir como elemento indispensable el recurso a una ley científica. Si trasladamos esto al modo en que un modelo científico puede proporcionar una explicación de un fenómeno, tendríamos entonces que concluir que sólo los modelos que contestan a preguntas por-qué y que contienen leyes, o que son ellos mismos leyes de cierto tipo, o que pueden generar argumentos que contienen leyes como premisas, podrían ser considerados como genuinamente explicativos. Sin embargo, si asumimos, con la mayoría de los filósofos de la biología, la inexistencia (o escasez) de leyes en la biología, ${ }^{3}$ entonces deberíamos aceptar asimismo que esta ciencia apenas tiene capacidad para proporcionar explicaciones. Y, no obstante, lo que observamos es que muchos modelos biológicos que no contienen leyes (biológicas), ni siquiera en un sentido lato, son considerados habitualmente como explicativos. ${ }^{4}$

1 Como es bien sabido, Wesley C. Salmon sostuvo que una explicación científica no adopta la forma de un argumento, sino que más bien ha de especificar los mecanismos causales que producen el fenómeno, o al menos los factores causales relevantes que lo producen. Esta forma de concebir la explicación científica encaja bastante bien con un cierto tipo de explicación mediante modelos (la explicación mecanicista) que ha sido prolijamente desarrollada en los últimos años, como diremos después. Uno de los principales problemas de este enfoque, sin embargo, es que no todos los modelos explicativos en biología son causales, como por ejemplo muchos modelos en genética de poblaciones (cf. Plutinski 2004). También Bas van Fraassen rechazó la exigencia de que una explicación científica haya de ser un argumento.

2 Mencionemos solo los nombres de Michael Scriven, Paul Humphreys, Wesley Salmon, Philip Kitcher, Bas van Fraassen, Nancy Cartwrihgt y James Woodward. Éste último, sin embargo, sostiene que una explicación debe contener, si no una ley estricta, sí al menos una generalización que permanezca invariable bajo algunas intervenciones (cf. Woodward 2001).

3 La literatura sobre la (in)existencia de leyes en la biología es demasiado amplia para ser incluida aquí. Algunos artículos influyentes son Beatty (1995), Brandon (1997), Mitchell (1997), Sober (1993 and 1997), Waters (1998), and Rosenberg (2001). Puede encontrarse una revisión del tema en el capítulo 6 de Diéguez 2012.

4 Podría argüirse que el modelo de van Frasssen -que no exige que la explicación sea un argumento ni que haga uso de una ley científica- podría ser adaptado para describir cómo explican los modelos biológicos. Para van Fraassen, una explicación es fundamentalmente una respuesta a una pregunta por-qué, que está especificada contextualmente, que hace uso de alguna teoría científica como conocimiento de fondo. Sin embargo, creo que éste no es el mejor camino a tomar. El modelo pragmático de explicación de van Fraassen sigue siendo demasiado restrictivo. En primer lugar, no todo modelo es una respuesta a una pregunta por-qué (vide infra). En segundo lugar, los modelos no son siempre capaces de establecer una clase de contraste en cualquier asunto. Y, finalmente, los criterios que van Fraassen propone para evaluar las respuestas dadas en una explicación establecen condiciones muy estrictas -tal como favorecer una clase de contraste en lugar de otra- que muchos modelos explicativos no pueden satisfacer. 
Incluso si -siguiendo en ello a Bogen (2005) y a Leuridan (2010)-admitimos que ciertos tipos de modelos (los modelos mecanísitcos), incorporan de algún modo leyes causales (biológicas) genuinas, aunque no sean regularidades universales estrictas (cf. Mitchell 1997), es dudoso que pueda exigirse este mismo requisito a muchos modelos biológicos importantes y significativos, como el modelo de bicapa lipídica de la membrana celular, o a modelos no mecanísticos.

Una posibilidad que debe ser considerada, y que goza de cierta popularidad, es la debilitar los requisitos del modelo de explicación por cobertura legal pero manteniendo su estructura argumentativa. En tal sentido, un modelo explicaría un fenómeno si es posible derivar de dicho modelo, junto con algunas premisas auxiliares, un enunciado que describa el fenómeno en cuestión, aunque sea en forma idealizada. En este caso, el modelo no se identificaría con una ley o con un grupo de leyes. Si el modelo consiste, por ejemplo, en un conjunto de ecuaciones (y, obviamente, no toda ecuación es la expresión de una ley), el fenómeno quedaría explicado si dichas ecuaciones permiten calcular los datos fundamentales que lo caracterizan. Desafortunadamente, esta alternativa atribuye a los modelos algunos rasgos que son controvertidos. En particular, identifica los modelos con entidades lingüísticas cuyos «mecanismos» explicativos serían fundamentalmente relaciones lógicas entre enunciados. Por otro lado, hay ciertos tipos de modelos, como por ejemplo los modelos icónicos (doy por sentado que este tipo de modelos proporcionan también explicaciones), cuya capacidad explicativa no se basa ciertamente en las consecuencias lingüísticas que pueden ser inferidas a partir de ellos, sino más bien en las relaciones perceptivas y estructurales entre sus elementos. Y finalmente, como pusieron de relieve los críticos del modelo de explicación por cobertura legal, muy a menudo las derivaciones lógicas o los cálculos no son suficientes para explicar un fenómeno. Como señala Carl Craver, «subsumir meramente un fenómeno bajo un conjunto de generalizaciones o bajo un modelo abstracto no es suficiente para explicarlo. [...] Los modelos ptolemaicos del sistema solar permiten predecir la localización de planetas en el cielo nocturno, pero nadie cree que el elaborado sistema de epiciclos, deferentes, ecuantes y excéntricas explique realmente por qué se mueven los planetas. Se puede usar la fórmula de Balmer para calcular las longitudes de onda del espectro de emisión del hidrógeno, pero la fórmula de Balmer no explica por qué las líneas espectrales aparecen donde lo hacen.» (Craver 2006, p. 358).

Después de esto, sólo parece quedar una alternativa que, como se ha dicho, cuenta ya con notables antecedentes y que algunos han vuelto a defender: la de «abandonar la conexión entre leyes y explicación teórica» (Cooper 1996). Creo que esta alternativa es la que se impone si hemos de dar cabida al modo en que explican los modelos científicos, dado que muchos de ellos no contienen leyes 
ni son convertibles en leyes. Pero entonces, la pregunta sigue en el aire: ¿en qué consiste explicar mediante un modelo?

\section{LA PLURALIDAD DE LOS MODELOS (Y DE SUS USOS)}

Entre las dificultades que encontramos para poder articular una respuesta a esta cuestión, sin duda una de las más importantes reside en la polisemia del término 'modelo'. En biología en particular, 'modelo' puede designar cosas muy diferentes: organismos concretos (como Drosophila melanogaster) que son ampliamente usados en investigación por sus especiales cualidades de fácil manejo o por ser representativos de otros organismos; objetos materiales que representan de forma simplificada a otras entidades (como los modelos moleculares hechos con plástico y metal); soluciones paradigmáticas a problemas empíricos (como el modelo de operón de la lactosa); interpretaciones teóricas e idealizadas de la estructura y mecanismos funcionales de algunas entidades o procesos biológicos (como el modelo de bicapa lipídica, el modelo llave-cerradura de la acción enzimática, el modelo de McArthur y Wilson de la biogeografía insular, el modelo de Mitchell de fosforilación oxidativa); conjunto de ecuaciones que describen algunos aspectos del comportamiento de sistemas biológicos complejos (como el modelo Lotka-Volterra de competencia interespecífica, el modelo cinemático Michaelis-Menten para la determinación de la velocidad de la acción enzimática, o el modelo metapoblacional de Levins); programas de simulación en ordenador (como los programas de Vida Artificial); etc.

Obviamente, no todo modelo ha sido concebido para explicar un fenómeno. En ocasiones, como en el caso de los modelos a escala, su función es meramente ilustrativa o ejemplificadora de los rasgos fundamentales de otra entidad. Y no son la explicación y la ejemplificación las únicas funciones que cumplen los modelos en la ciencia. También pueden ser usados como elementos auxiliares en la experimentación, la manipulación o la enseñanza; o como fuente de razonamientos subrogatorios; ${ }^{5}$ como heurísticos para el logro de nuevas hipótesis o para la orientación en el análisis de escenarios alternativos posibles; o como herramientas para la predicción; o como instrumentos para el cálculo o para la precisión de ideas; o como meras descripciones simplificadas de estructuras y procesos; o como pruebas de la posibilidad de existencia, etc.

Demetris Portides $(2008$, p. 385) ha señalado que la mayor parte de estos sentidos de ‘modelo’ están ligados a las ideas de representación e idealización. Pero incluso este mínimo común denominador puede ser llevado a cabo de maneras muy diferentes (cf. Morrison 1999 and Weisberg 2007b). ¿Puede en-

5 Esta función ha sido subrayada por diversos autores como central en el uso de los modelos en la ciencia (Swoyer 1991, Suárez 2004, Contessa 2007, y de Donato y Zamora Bonilla 2009). Coincido ampliamente con esta tesis. 
contrarse, entonces, algún elemento común a las explicaciones proporcionadas por estos diversos tipos de modelos? Creo que sí, que hay un modo de ofrecer una respuesta afirmativa a esta pregunta. Por supuesto que con ello no pretendo ofrecer nada parecido a una teoría general de la explicación mediante modelos. No estoy muy seguro de que algo así sea factible, dada precisamente la polisemia del término. Me limitaré simplemente a señalar un rasgo común del uso explicativo de los modelos que merece ser explorado.

En general, puede afirmarse que, tanto en la ciencia como en la vida cotidiana, se logra una explicación de un hecho cuando este hecho es incorporado a un cuerpo previo de conocimientos mediante conexiones que muestren que el hecho es causalmente posible dado ese cuerpo de conocimientos. La concepción heredada de la explicación científica capturaba esta intuición mediante la idea de que pedir una explicación científica de un fenómeno era equivalente a preguntar por qué sucedió el fenómeno. Sin embargo, es fácil ver que muchos modelos en la ciencia no pueden interpretarse como buscando respuestas a preguntas por-qué (why-questions), sino más bien a preguntas cómo-es-posiblemente (howpossibly-questions), cómo-es-de-hecho (how-actually) y cómo-es-plausibleque-sea (how-plausibly) (cf. Salmon 1989, pp. 136-138, Machamer, Darden \& Craver 2000, Plutynski 2005, and Craver 2006). El conocido 'modelo estándar' en cosmología, por ejemplo, explica cómo comenzó posiblemente el Universo, no por qué comenzó. El modelo de bicapa lipídica para la membrana celular explica cómo plausiblemente dicha membrana permite el flujo de ciertas sustancias y no de otras, no por qué funciona de ese modo. El modelo cinemático de Michaelis-Menten explica cómo de hecho la velocidad de las reacciones enzimáticas varía en ciertas circunstancias, no por qué lo hace así.

Por ello, propongo entender la explicación científica en un sentido amplio como una respuesta a una pregunta (no necesariamente a una pregunta por-qué) suscitada en un contexto científico que proporciona una mejor comprensión de algún fenómeno o conjunto de fenómenos. Esta definición amplia permite incluir fácilmente a los modelos como herramientas explicativas. Si hay algo en común en el modo en que explican los diferentes tipos de modelos es esto: la explicación aumenta nuestra comprensión del sistema representado por el modelo. Ciertamente, esta conexión entre explicación y comprensión no es nada nuevo. Frente a la tradición historiográfica antipositivista decimonónica y a la filosofía hermenéutica, que ha tendido a contraponer la explicación científica a la comprensión de acciones y significados, son muchos los autores que han establecido una fuerte conexión entre ambos conceptos (cf. Scriven 1962, Friedman 1974, Kitcher 1981, Achinstein 1983, Kim 1994, Salmon 1998, Faye 1999).

En la filosofía de las ciencias sociales, ya desde los trabajos de Droysen y Dilthey, ha sido habitual contrastar la comprensión y la explicación como objetivos metodológicos diferentes e incluso opuestos. Las corrientes historicistas 
y hermenéuticas han sostenido que mientras que las ciencias naturales deben buscar la explicación causal de los fenómenos, las ciencias sociales deben, en cambio, buscar prioritariamente la comprensión del significado de las acciones humanas. De acuerdo con ello, comprender es captar un significado (de alguna acción humana o de alguna intención). Este procedimiento metodológico ha de estar, pues, restringido a las ciencias sociales, porque no hay lugar para los significados en el ámbito de los objetos naturales. El comportamiento de las entidades físicas no parece tener significado alguno. Sin embargo, cabe aducir que este enfoque asume un sentido muy estrecho del término 'comprensión'. No sólo los productos o las actitudes humanas (acciones, intenciones, inferencias, ideas, obras de arte, discursos, etc.) son susceptibles de comprensión. También pueden ser comprendidas las situaciones objetivas, las funciones, los mecanismos de funcionamiento, las relaciones entre cosas, etc., y todo ello puede ser localizado en entidades físicas y biológicas (cf. Salmon 1998). Podemos tener un conocimiento comprensivo no sólo acerca del significado de una acción sino también acerca de los mecanismos homeostáticos que mantienen constante la temperatura en los mamíferos, o acerca de los procesos de autorreplicación del ADN y de las razones por las que es semiconservativa. Por lo tanto, pese a lo que han defendido diversos autores de la tradición hermenéutica, es posible también la comprensión en el ámbito de los fenómenos naturales.

Si nos centramos en los modelos en lugar de en las teorías, parece haber diversos modos en los que éstos pueden mejorar nuestra comprensión de los fenómenos. Una de las formas más importantes, y posiblemente la más frecuente, en las que pueden hacerlo es describiendo, de una forma simplificada, los mecanismos causales que producen el fenómeno y la interacción de sus componentes. Éste es, con diferencia, el caso más estudiado en la literatura reciente sobre el tema (cf. Salmon 1998, p. 77, Machamer, Darden \& Craver 2000, Bechtel \& Abrahamsen 2005, Craver 2006, Darden 2008, Bechtel 2011). Pero no debería pensarse que esta es la única forma en la que lo hacen. Otra forma en la que mejoran nuestra comprensión es poniendo de relieve por medio de ecuaciones matemáticas las relaciones estructurales entre los rasgos fundamentales del fenómeno representado. Otra es mediante la unificación de fenómenos constitutivamente diferentes. Otra, como en el caso de la «explicación por equilibrio» proporcionada por modelo de Fisher de proporción de sexos, es mostrando cómo diversos escenarios causales con diferentes condiciones iniciales conducen sin embargo al mismo resultado. Otra, ejemplificando propiedades del sistema representado (cf. Elgin 2009). Otra, ofreciendo un «mundo creíble» que presente ciertas similitudes importantes con el mundo real (cf. Sudgen 2011). Y a buen seguro podrían señalarse bastantes más formas. En este asunto, como en tantos en filosofía de la biología, el enfoque pluralista parece inevitable (cf. Plutynski 2004, Bokulich 2011, Brigandt 2011). 


\section{OBJETIVANDO LA COMPRENSIÓN}

A nadie se le oculta, sin embargo, que la propuesta que acabo de hacer tiene sus puntos débiles. Así, y a pesar de la importancia de este asunto, el análisis preciso de la noción de comprensión científica en relación con los fenómenos naturales está aún en sus comienzos. Y si quiere ser de alguna utilizad para la clarificación de la noción de explicación, ha de enfrentarse a serias dificultades conceptuales y teóricas. Entre ellas no es la menor la que hace años ya señalara Hempel (1965, pp. 425-6), a saber: que la comprensión es un asunto meramente subjetivo, una noción atribuible sólo a estados subjetivos de individuos concretos, y por tanto un concepto relativo y no generalizable. Un sentido puramente subjetivo de comprensión carecería de valor epistémico suficiente como para servir de base a la noción intersubjetiva de explicación científica, puesto que lo que fuera comprensible para una persona en este sentido podría no serlo para otra. A tal respecto, de Recht, Leonelli y Eigner (2009, p. 3) han señalado que «hay una diferencia importante entre comprensión y explicación: mientras que se puede decir legítimamente que la teoría T explica el fenómeno F, solo se puede hablar de la comprensión de F por medio de T si se invoca a un sujeto (en este caso al científico). En otras palabras, mientras que la explicación puede ser vista como una relación binaria entre un explanans (por ejemplo, una teoría) y un explanandum (el fenómeno), la comprensión es siempre una relación ternaria que implica explanans, explanandum y sujeto». ${ }^{6}$

El problema, por tanto, consiste en averiguar si hay algún criterio para distinguir entre la mera sensación de comprensión y la comprensión genuina o si, por el contrario, la referencia necesaria a un sujeto convierte a la comprensión en algo irremediablemente subjetivo. Éste es, de hecho, un viejo problema: fue la crítica principal que recibió el método de Verstehen en el debate clásico en metodología de las ciencias sociales.

Ciertamente, es imposible proporcionar un conjunto exhaustivo y permanente de criterios que permita señalar cuándo estamos ante una comprensión genuina, puesto que estos criterios poseen elementos contextuales que han cambiado a lo largo de la historia de la ciencia (de Regt \& Dieks 2005). No obstante, creo que pueden ofrecerse algunas indicaciones útiles que permitan decidir de forma razonable si un modelo científico nos proporciona o no una comprensión genuina. Por razones de espacio, aquí sólo podré presentar algunas

6 Schurz y Lambert (1994, p. 67) también caracterizan la comprensión como una relación ternaria, pero, en su intento de evitar el subjetivismo, consideran que esta relación es entre una respuesta, un fenómeno y un corpus cognitivo. Básicamente su propuesta es que una respuesta a una «pregunta-cómo en pos de una comprensión» contribuye a la comprensión de un fenómeno si y sólo si muestra cómo el fenómeno encaja en el corpus cognitivo. Esta propuesta, en mi opinión, es sin embargo demasiado genérica. 
de dichas indicaciones, sin pretender justificarlas en detalle. Para ello hemos de comenzar por distinguir entre dos tipos diferentes de modelos a los que denominaré 'modelos contrastantes' y 'modelos representativos'.

Entiendo por modelos contrastantes aquéllos construidos intencionalmente como recursos explicativos extremadamente no-realistas, como es el caso de los modelos falsos en biología (e.g. poblaciones con más de dos sexos, poblaciones con herencia mezclada en lugar de mendeliana). Son modelos que no tratan de representar ningún sistema real y que no se hacen más precisos mediante un proceso de des-idealización. El sistema representado es completamente ficticio, aunque es propuesto para entender algunos aspectos de un sistema-diana real.En este tipo de modelos, la carga explicativa reside justamente en las disimilitudes que el modelo presenta con el sistema-diana.

Para este tipo de modelos, propongo un criterio cualitativo simple de comprensión genuina:

Un modelo contrastante nos proporciona una comprensión genuina del comportamiento de un sistema real si el contraste entre las consecuencias derivables del modelo y el sistema-diana real permite revelar cómo algunas características interesantes del comportamiento del sistema real dependen de la presencia o ausencia de ciertas circunstancias que están ausentes o presentes respectivamente en el modelo.

Por modelos representativos entiendo aquellos que encajan de algún modo con el sistema-diana, o, en otros términos, aquellos que son construidos con el propósito de representar algunos aspectos de los fenómenos presentes en un sistema real.

Para este tipo de modelos, dada su variedad, es más difícil seleccionar criterios generales que sirvan como indicadores de comprensión genuina. No obstante, creo que los siguientes, muy básicos y puramente negativos, pueden ser una base mínima que permita excluir algunos modelos por no cumplir su función:

Un modelo representacional no proporciona una comprensión genuina del sistema-diana si:

1. Las analogías entre el modelo y el sistema-diana son débiles, ad hoc, o científicamente infundadas.

2. Formula abstracciones sobresimplificadas que excluyen factores funcionales relevantes, esto es, factores que son necesariamente constitutivos del comportamiento del sistema modelado.

3. Realiza idealizaciones extremadamente irreales o inútiles, esto es, idealizaciones que están tan alejadas de las condiciones con las que ha 
de enfrentarse el sistema modelado que, incluso tras correcciones progresivas, no ayudan a ver cómo este sistema varía en las circunstancias usuales bajo algunas manipulaciones.

4. Postula una ontología fantástica o pseudocientífica.

5. Los mecanismos postulados carecen de analogías con los mecanismos que funcionan en el sistema real.

6. Sus predicciones adicionales fracasan sistemáticamente.

\section{CONCLuSIONES}

Los modelos científicos son recursos explicativos fundamentales en la ciencia, y particularmente en aquellas ciencias en las que es dudoso que podamos contar con leyes científicas genuinas, como es el caso de la biología (y de las ciencias sociales). La cuestión de cómo explican los modelos ha despertado una gran atención en las últimas décadas y, sin embargo, sigue siendo una cuestión controvertida. Hay muchos tipos de modelos y no es de extrañar, por tanto, que puedan proporcionar explicaciones de los fenómenos de formas muy diversas. Si se puede señalar un rasgo común a todos estos modos diferentes de explicar, es el hecho de que los modelos nos ofrecen una mejor comprensión de los fenómenos. Se ha objetado que la noción de ‘comprensión' es irremediablemente subjetiva. Sin embargo, pueden proporcionarse algunos criterios contextuales pero objetivamente justificables para decidir cuándo un sentimiento de comprensión corresponde a una comprensión genuina. Estos criterios no pueden eliminar por completo un cierto grado de subjetivad en la comprensión de un fenómeno proporcionada por un modelo, pero sería desmesurado esperar algo así. Al fin y al cabo, incluso en la ciencia, siempre cabe que lo que un científico considera una explicación satisfactoria no sea visto del mismo modo por otro científico.

\section{REFERENCIAS}

ACHINSTEIN, P. (1983), The Nature of Explanation. New York: Oxford University Press.

BARTELBORTH, T. (2002), «Explanatory Unification». Synthese, 130, 91-107.

BEATTY, J. (1995), «The Evolutionary Contingency Thesis», en G. Wolters and J. Lennox (Eds.), Concepts, Theories, and Rationality in the Biological Sciences. Pittsburgh: University of Pittsburgh Press, pp. 83-97.

BECHTEL, W. (2011), «Mechanism and Biological Explanation». Philosophy of Science. Vol. 78, No. 4, pp. 533-557.

BECHTEL, W. \& ABRAHAMSEN, A. (2005), «Explanation: A Mechanist Alternative». Stud. Hist. Phil. Biol. \& Biomed. Sci., 36, pp. 421-441. 
BOGEN, J. (2005), «Regularities and causality; generalizations and causal explanations». Stud. Hist. Phil. Biol.\& Biomed. Sci., 36, pp. 397-420.

BOKULICH, A. (2011), «How Scientific Models Can Explain». Synthese, 180 (1), 33-45.

BRAITHWAITE, R. B. (1953), Scientific explanation; a study of the function of theory, probability and law in science. New York, NY: Cambridge University Press.

BRANDON, R. N. (1997), «Does Biology Have Laws? The Experimental Evidence». Philosophy of Science, 64 (proceedings), 444-457

BRIGANDT, I. (2011), «Explanation in Biology: Reduction, Pluralism and Explanatory Aims». Science and Education. (Online first). doi:10.1007/s11191-011-9350-7

CARTWRIGHT, N. (1983), How the Laws of Physics Lie. (Oxford: Clarendon Press)

CONTESSA, G. (2007), «Scientific Representation, Interpretation, and Surrogative Reasoning». Philosophy of Science, 74, 48-68.

COOPER, G. (1996), «Theoretical Modeling and Biological Laws». Philosophy of Science, 63, S28-S35

CRAVER, C.F. (2006), «When Mechanistic Models Explain». Synthese, 153, pp. 355 376.

DARDEN, L. (2008), «Thinking Again about Biological Mechanisms». Philosophy of Science, 75 , pp. 958-969.

De DONATO, X. \& ZAMORA BONILLA, J. (2009), «Credibility, Idealization, and Model Building: An Inferential Approach». Erkenntnis, 70, 101-118.

De REGT, H.W. \& DIEKS, D. (2005), «A Contextual Approach to Scientific Understanding». Synthese, 144, pp. 137-170.

De REGT, H. W., LEONELLI, S. \& EIGNER, K. (2009), «Focusing on Scientific Understanding», en H. W. de Regt, S. Leonelli \& K. Eigner (Eds.) Scientific Understanding: Philosophical Perspectives Pittsburgh: University of Pittsburgh Press, pp. 1-17.

DIÉGUEZ, A. (2012), La vida bajo escrutinio. Barcelona: Biblioteca Buridán.

DÍEZ, J. A. (2002), «Explicación, unificación y subsunción teórica», en W. González (Ed) Pluralidad de la explicación científica Barcelona: Ariel, pp. 73-93.

ELGIN, C. Z. (2009), «Is Understanding Factive?», en D. Pritchard, A. Millar \& A. Haddock (Eds.) Epistemic Value Oxford: Oxford University Press, pp. 322-329.

ELGIN, M. \& SOBER, E. (2002), «Cartwright on Explanation and Idealization», Erkenntnis, 57, 441-450.

FAYE, J. (1999), «Explanation Explained», Synthese, 120, pp. 61-75.

FORGE, J. (2002), «Reflections on Structuralism and Scientific Explanation». Synthese, $130,109-121$

FRIEDMAN, M. (1974), «Explanation and Scientific Understanding», The Journal of Philosophy, 71, pp. 5-19.

FRIGG, R. (2006), «Scientific Representation and the Semantic View of Theories», Theoria 55, 49-65.

HEMPEL, C.G. (1965), Aspects of Scientific Explanation and Other Essays in the Philosophy of Science. New York: Free Press.

HESSE, M. (1966), Models and Analogies in Science. (Oxford, Oxford University Press). 
KIM, J. (1994), «Explanatory Knowledge and Metaphysical Dependence», Philosophical Issues, 5, pp. 51-69.

KITCHER, Ph. (1981), «Explanatory Unification». Philosophy of Science, 48, pp. 507-531.

LEONELLI, S. (2009), «Understanding in Biology: The Impure Nature of Biological Knowledge», en H. W. de Regt, S. Leonelli \& K. Eigner (Eds.) Scientific Understanding: Philosophical Perspectives Pittsburgh: University of Pittsburgh Press, pp. 189-209.

LEURIDAN, B. (2010), «Can Mechanisms Really Replace Laws of Nature?». Philosophy of Science, 77, 317-340.

MACHMER,P.K., DARDEN,L., CRAVER,C.F.(2000), «Thinking about Mechanisms». Philosophy of Science, 57, pp. 1-25.

McMULLIN, E. (1978), «Structural Explanation». American Philosophical Quarterly, $15,139-147$.

MITCHELl, S. D. (1997), «Pragmatic Laws». Philosophy of Science, 64 (proceedings), 468-479

MOULINES, C. U. (2005), «Explicación teórica y compromisos ontológicos: un modelo estructuralista». Enrahonar, 37, 45-53

NAGEL, E. (1961), The Structure of Science. Problems in the Logic of Scientific Explanation. New York: Harcourt, Brace and World.

PLUTINSKI, A. (2004) «Explanation in Classical Population Genetics». Philosophy of Science, 71, 1201-1214.

--- (2005), «Explanatory Unification and the Early Synthesis». British Journal for the Philosophy of Science, 56, 596-609

ROSENBERG, A. (2001), «How is Biological Explanation Possible?». British Journal for the Philosopy of Science, 52, 735-760

SALMON, W. (1989), «Four Decades of Scientific Explanation», en Ph. Kitcher and W. Salmon (eds.) Scientific Explanation. Minnesota Studies in the Philosophy of Science, vol. XIII, Minneapolis: University of Minnesota Press, pp. 3-219.

SCHURZ, G. \& LAMBERT, K. (1994), «Outline of a Theory of Scientific Understanding». Synthese, 101, 65-120.

SCRIVEN, M. (1962), «Explanations, Predictions, and Laws», en H. Feigl and G. Maxwell (eds.) Scientific explanation, space, and time. Minneapolis: University of Minnesota Press, pp. 170-230.

SOBER, E. (1983), «Equilibrium Explanation». Philosophical Studies, 43, 201-210. , (1993), Philosophy of Biology. (Boulder: Westview).

, (1997), «Two Outbreaks of Lawlessness in Recent Philosophy of Biology». Philosophy of Science, 64 (proceedings), 458-467.

SUÁREZ, M. (2004), «An Inferential Conception of Scientific Representation». Philosophy of Science, 71, 767-779

SUDGEN, R. (2011), «Explanations in search of Observations». Biology and Philosophy, 26, pp. 717.736

SWOYER, C. (1991), «Structural Representation and Surrogative Reasoning». Synthese, $87,449-508$. 
WOODWARD, J. (2001), «Law and Explanation in Biology: Invariance is the Kind of Stability That Matters». Philosophy of Science, 68, 1-20.

Antonio Diéguez es Catedrático de Lógica y Filosofía de la Ciencia en el Departamento de Filosofía de la Universidad de Málaga (España).

Líneas de investigación:

Epistemología evolucionista, realismo científico, filosofía de la tecnología, filosofía de la biología.

Publicaciones recientes:

(2012), La vida bajo escrutinio. Una introducción a la filosofía de la biología, Barcelona: Biblioteca Buridán.

(2013) «Life as a Homeostatic Property Cluster», Biological Theory, Volume 7, Issue 2, Page 180-186.

http://www.springerlink.com/openurl.asp?genre=article\&id=doi:10.1007/s13752-012$0052-4$

Dirección electrónica: dieguez@uma.es 